\title{
Challenges in Telemedicine: Even When the Road is Hard, Never Give up
}

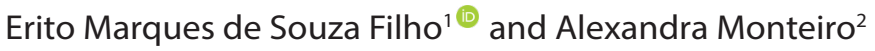

Universidade Federal Rural do Rio de Janeiro,' Rio de Janeiro, RJ - Brazil

Universidade do Estado do Rio de Janeiro, ${ }^{2}$ Rio de Janeiro, $R J$ - Brazil

Editorial referring to the article: Implementation of a Telecardiology Service in a Health Unit in the City of Porto Alegre, Brazil: A Pilot Study

"Even when the road is hard, never give up" Tupac Shakur and The Outlawz

The above quote is from the hit song "Baby Don't Cry" immortalized by Tupac and The Outlawz. The song is about a young woman who faces challenges and receives encouragement to persevere and not give up. In a similar way, telemedicine faces several challenges on an arduous path. In this way, imbued with a tenacious spirit, its users have had remarkable experiences. In the current issue of the International Journal of Cardiovascular Sciences, Esmerio and colleagues presented a successful application of a telecardiology service. They emphasized that the work contributed to reducing the distance between patients and specialized professionals, and they highlighted a high level of satisfaction for both patients and health Professionals. ${ }^{1}$ Experiences like this represent the relevant role of telemedicine in providing quality health services and generating value. However, these tools still face important challenges that need to be overcome.

The first challenge is resistance. Some people underestimate the potential of telemedicine, arguing that it damages the doctor-patient relationship and can harm patients. That is not true per se; however, using telemedicine without respecting the ethical dictates of the medical profession would be disastrous. In this context, a fundamental point emerges in order to reduce resistance. It is necessary to increase patients' and health professionals' literacy and to design studies that

\section{Keywords}

Cardiovascular Disease; Telemedicine/ethics, Telemedicine/methods; Telemedicine/trends; Bioethic; Telehealth; Women; Working; Distance Education; Brazil. demonstrate its real benefits in clinical practice, such as the successful example mentioned above. Literacy is equally important in relation to the use of computing resources to access telemedicine tools, which must be user-friendly. Almufleh and colleagues, for example, highlight the need to include telemedicine training in the medical curriculum. They reiterate three fundamental components of a telemedicine curriculum: the issue of technology per se; examination and communication skills; and the medicolegal/licensing aspects. ${ }^{2}$

The second challenge concerns the limits of telemedicine. It is necessary to specify, from the outset, what kind of benefits can be provided to patients. If, for example, during an appointment, a patient reports chest pain with characteristics of an acute myocardial infarction, it is mandatory for the patient to go to the emergency room for clinical evaluation and complementary tests to rule out or confirm the condition. In this hypothetical case, the teleconsultation did not resolve the complaint; nevertheless, it was fundamental in signaling the need for emergency care. We clearly must not attempt to go beyond what we can deliver. Furthermore, telemedicine is complementary to face-to-face care, and it does not represent a replacement; it must be practiced safely and for a period that is compatible with what the clinical circumstances require. ${ }^{3}$ It is also necessary to respect limits and explain to the patient that in-person consultation may be necessary. Accordingly, the request to sign a free and informed consent form plays a fundamental role.

The third challenge deals with data security and reliability of electronic systems. This should be a constant concern, as data leakage and access by unauthorized persons could have catastrophic effects on patients' lives, including worsening their health issues. Specific legislation on the subject has brought great progress, but there is still

Mailing Address: Erito Marques

Universidade Federal Rural do Rio de Janeiro

Rua Governador Roberto da Silveira, w/n. Postal Code 26285-060, Nova Iguaçu, RJ - Brazil

E-mail: mederitomarques@gmail.com

DOI: https://doi.org/10.36660/ijcs.20220014 
a long way to go. In addition, it is worth mentioning that the data should be of good quality (healthy data). ${ }^{4}$

The fourth major challenge refers to the relationship between telemedicine and other digital tools. It is necessary to understand that artificial intelligence, smartphone apps, electronic health records, blockchain, wearables, and others constitute a continuum of solutions that must be considered in an integrated manner and aligned with the purpose of generating value. In addition, it is essential for telemedicine activities to be properly integrated into the practice of the multidisciplinary health team. ${ }^{5}$ The main idea is build a sustainable digital ecosystem. If, on the one hand, telemedicine tools have been used successfully in different contexts and are capable of overcoming distances, on the other hand, a significant portion of the Brazilian population cannot yet access these benefits. Thus, the fifth challenge is to expand patients' access to these tools. This implies several needs, such as expansion of infrastructure and the development of customized management models.

\section{References}

1. Esmerio FG, Goldmeier S, Barbosa ECD, Segredo LM, Silva R, Irigoyen $\mathrm{MC}$ et al. Implementation of a telecardiology service in a health unit in the city of Porto Alegre, Brazil: A Pilot Study. Int J Cardiovasc Sci. 2022; 35(2):152-158. Doi: https://doi.org/10.36660/ijcs.20200305.

2. Almufleh A, Lee C, Tsang MY, Gin K, Tsang TSM, Nair P. The Need for telemedicine integration into adult cardiology training Curricula in Canada. Can J Cardiol. 2021 Jun;37(6):929-32. doi: 10.1016/j. cjca.2021.03.001.

3. Lopes MACQ, Oliveira GMM, Ribeiro ALP, Pinto FJ, Rey HCV, Zimerman LI, et al. Guideline of the Brazilian Society of Cardiology on Telemedicine
The sixth challenge is about the need for continuous evaluation of the tools and the development of evaluation metrics. In the meantime, the work by Esmerio and colleagues ${ }^{1}$ exposes something fundamental: the need to assess the degree of satisfaction of the patients for whom the tool was designed. In addition, it is important to validate these tools in different contexts and to encourage sharing of experiences. Last but not least, patient engagement with telemedicine care might help address unmet health needs by more accurately identifying them. In this context, it is important to design more tailored and people-centered services. All this would not only help to cut wasteful spending; more importantly, it would result in healthier and more productive populations.

Resistance, limits to use, security and reliability of systems, the relationship with other digital tools, access, continuous evaluation, and engagement are important challenges to be overcome and must be the object of efforts on the part of the entire community. Surely, it is an arduous task. However, we can quote Tupac again: "Even when the road is hard, never give up".

in Cardiology - 2019. Arq Bras Cardiol. 2019 Nov;113(5):1006-56.1056. doi: 10.5935/abc.20190205. PMID: 31800728

4. Souza Filho EM, Fernandes FA, Soares CLA, Seixas FL, Santos AASMDD, Gismondi RA, et al. Artificial intelligence in cardiology: concepts, tools and challenges - "The Horse is the One Who Runs, You Must Be the Jockey". Arq Bras Cardiol. 2020 Apr;114(4):718-25.doi: 10.36660/abc.20180431.

5. Monteiro A, Grisolia BM, Aguiar J, Santos M. Profile and motivation of women enrolled in the brazilian telemedicine and telehealth postgraduation distance education programme. J Int Soc Telemed eHealth.2019;7:e3-1-e3-4. DOI: https://doi.org/10.29086/JISfTeH.7.e3. 